\title{
O CONTEXTO DO DESIGN INCLUSIVO EM PROJETOS DE PRODUTO: ENSINO, PRÁTICA E ACEITAÇÃO
}

Danila Gomes

LEUI | Laboratório de Ergodesign e Usabilidade de Interfaces

PUC-Rio, Brasil

danilagomespe@gmail.com

Manuela Quaresma

LEUI | Laboratório de Ergodesign e Usabilidade de Interfaces

PUC-Rio, Brasil

mquaresma@puc-rio.br

Resumo: O presente artigo é resultado de uma pesquisa bibliográfica de uma dissertação de mestrado, onde foram explorados artigos, trabalhos, dissertações e teses provenientes de diversos lugares do mundo com o intuito de entender o contexto do Design Inclusivo (DI) no meio acadêmico, no mercado profissional e na indústria. O DI, também conhecido como Design Universal e Design para todos (Design for all) é uma abordagem de projeto que beneficia a diversidade humana através de produtos mais eficientes para uma gama maior de usuários, sejam pessoas com limitações atípicas ou não. Essa prática de projeto vem sendo fomentada em vários países, não obstante, ainda são escassos produtos inclusivos no mercado. Neste artigo consta alguns fatores observados diante deste contexto. São trazidas à tona algumas questões, tais como: I) as dificuldades de aplicação, ou seja, os obstáculos na transposição da teoria à prática de projeto; e II) as barreiras entre o Design Inclusivo e a indústria, isto é, as impedições na transformação do Design Inclusivo em um produto rentável. Partindo do princípio que a prática é resultado do aprendizado, um dos fatores que dificultam a realização dessa abordagem por profissionais é o não ensino do DI nas instituições. Do mesmo modo, o desconhecimento e a não aceitação dos diretores industriais são barreiras que impedem essa prática nas indústrias, ficando para o designer a incumbência de deixar claro que investir em produtos inclusivos, além de ser relevante para a inclusão social, é uma forma de conquistar mercados até então inexplorados e pode ser um caminho valioso para a inovação.

Palavras-chave: Design, Design Inclusivo, projeto de produto.

Abstract: This article is the result of a Masters dissertation literature review, in which articles, papers, dissertations and theses from various places worldwide were examined in an intent to understand the context of Inclusive Design (ID) in the academic circle, professional market, and industry. ID, also known as Universal Design and Design for All is a design approach which benefits human diversity through more efficient products 
to a bigger array of users; no matter if they have some atypical limitation or not. However, although the use of this approach has been fomented in many countries, inclusive products are still very scarce in the market. Some factors observed in this context are reported in this article. So, some questions have been explored, such as I) the difficulties of application, that is, the obstacles to transport the theory to the practice; and II) barriers between Inclusive Design and industry, in other words, any kind of limitation in transforming the Inclusive Design into a profitable product. Assuming that practice is a result of learning, one of the factors that can cause difficulties for professionals on executing this approach is when ID isn't taught in institutions. Likewise, the lack of knowledge and the disapproval by industry directors are obstacles, which prevent it to be introduced in industries. So, it becomes the designer's duty to make it clear that investing in inclusive products, besides of being significant for social inclusion, is an opportunity to conquer new markets, until unexplored, and also a valuable way to innovation.

Keywords: Design, Inclusive Design, product design.

\section{INTRODUÇÃO}

Design Inclusivo (DI), também conhecido como Design Universal e Design para todos (Design for all) é uma abordagem de projeto que busca incorporar a diversidade de uso, ou seja, objetiva considerar o maior número de pessoas possível na elaboração de produtos, serviços ou ambientes. Portanto, é uma filosofia a ser adotada na condução de todo o processo de desenvolvimento de um projeto.

A necessidade desta prática se dá na percepção da diversidade humana e na necessidade de considerá-la. A inclusão de pessoas com ou sem limitações atípicas na elaboração de objetos de uso comum a todos corrobora a igualdade de oportunidades e uma vida mais prática e independente. Apesar da necessidade de aplicação, a prática do DI não é difundida ou popularizada no Brasil. A importância do DI é nítida aos nossos olhos e o seu conceito permeia por países como os Estados Unidos, Inglaterra e Japão. Não obstante, há pouca difusão da sua prática em projetos elaborados por designers e produtos inclusivos continuam a ser exceção no mercado.

A não adoção dessa prática de projeto se sucede em diversos países. À vista disso, em alguns lugares estão estudando formas de facilitar a abordagem inclusiva para os designers, além de se preocuparem também com a aceitação de investimento na fabricação de produtos inclusivos. Portanto, algumas questões que envolvem a aplicabilidade do DI e a viabilidade de projetos inclusivos na indústria serão trazidas à tona neste artigo.

O presente artigo é oriundo de um levantamento bibliográfico sobre o DI. Neste levantamento, artigos, trabalhos, dissertações e teses provenientes de diversos lugares do mundo foram explorados almejando entender o contexto desta abordagem de projeto no meio acadêmico, no mercado profissional e na indústria. A seguir veremos o contexto do ensino e da prática do DI apreciado em uma pesquisa teórica que constitui parte da dissertação de mestrado nomeada como: A aplicabilidade do Design Inclusivo em projetos de produto no Brasil. 


\section{DIFICULDADES DE APLICAÇÃO}

Acredita-se que a dificuldade na aplicação do DI em projetos de produto está atrelada à formação e à prática dos designers. No Brasil não há uma difusão dessa abordagem de projeto que repercuta entre os novos designers. Entende-se que isso acontece pela pouca ênfase dada nos cursos de Design com foco em projeto de produto.

Esta hipótese foi levantada a partir de uma pesquisa exploratória com alguns professores de cursos de Design no Brasil, onde estes foram questionados quanto ao seu conhecimento sobre DI. Em suas respostas pôde-se perceber que alguns não o conheciam e nenhum deles ensinavam. Portanto, levando em consideração que a prática é resultado do aprendizado, cabe levantar que o não ensino do DI influencia diretamente na não aplicação deste em projetos profissionais.

\subsection{Um apanhado sobre o ensino e a difusão do Design Inclusivo}

A adoção dessa abordagem de projeto se deu mais nos cursos de Arquitetura, como Desenho Universal ou Design Universal (DU). Essa adoção se configura em formato de disciplina, cursos de extensão, de curta duração ou cursos de especialização, de longa duração com mais aprofundamento teórico e prático.

Segundo Dorneles et al. (2011, p.149) o ensino do DU nos cursos de Arquitetura no Brasil teve início na década de 1990 e as dificuldades iniciais encontradas pelos professores era a falta de material didático em português para repassar aos alunos e isso gerou, num primeiro momento a busca por desenvolver bibliografias nacionais. Segundo os autores, as primeiras bibliografias nacionais foram lançadas em 2001, como o relatório de pesquisa "Desenho Universal por uma Arquitetura inclusiva" desenvolvido por professores e alunos do curso de Arquitetura e Urbanismo da Universidade Federal de Santa Catarina.

Apesar da difusão do DI ser mais evidente na área da Arquitetura no Brasil, até pelo fato de estar presente em legislações e normas de arquitetura e urbanismo, acredita-se que é possível encontrar estudos pertinentes no vasto campo do Design. Portanto, ao decorrer da investigação, que se encontra em desenvolvimento, conduzida pelas autoras deste artigo, buscar-se-á, como parte crucial da pesquisa de mestrado, através de pesquisas de campo, identificar os locais onde estão ensinando o DI e a forma como este está sendo ensinado nos cursos de Design com ênfase em projetos de produto no país.

Com o intuito de ter uma visão panorâmica do ensino do DI no mundo, foram levantados os países que adotaram essa prática de projeto juntamente com os locais de pesquisa e estudo.

\section{Reino Unido}

No Reino Unido encontra-se o Grupo de Design Inclusivo do Engineering Design Centre localizado na Universidade de Cambridge formado por um grupo de pesquisadores, dentre eles professores e doutorandos, que investigam a interação entre as exigências que os produtos fazem a seus usuários e a variada gama de capacidades desses usuários. Este grupo de pesquisadores desenvolveu o Inclusive Design Toolkit; um conjunto de ferramentas para auxiliar a prática dessa abordagem de projeto. Dentre elas estão ferramentas de auditoria e simuladores wearable, para incentivar os designers a oferecerem produtos e serviços mais inclusivos. 
O Grupo de Design Inclusivo se constitui em uma das linhas de pesquisa do Engineering Design Centre que transfere conhecimentos através de ensino, de treinamentos, de publicações e de colaboração industrial. Uma importante ferramenta utilizada por este grupo é a 'Exclusion Calculator,' uma calculadora capaz de estimar a exclusão que um produto pode oferecer baseada nas seis capacidades-chave dos usuários, como: visão, audição, capacidade de pensar, destreza, capacidade de alcançar e capacidade de locomoção. Esta calculadora é uma ferramenta valiosa para demonstrar a quantidade de pessoas que estão sendo excluídas do uso de certos objetos, ou seja, a quantidade de consumidores inativos, que poderiam ser potenciais consumidores se a empresa fabricante adotasse a abordagem do DI.

O Centre Helen Hamlyn for Design (CHHD) do Royal College of Art localizado em Londres, é decorrente do programa de pesquisa-ação DesignAge, que teve início em 1991 e que se tornou um centro de pesquisa pleno em 1999, direcionando o seu foco para projetos que melhoram a vida das pessoas considerando uma série de necessidades sociais. (CHHD, 2015) O Centro desenvolve métodos de pesquisa e dissemina o conhecimento através do ensino (como cursos e workshops), eventos, publicações e colaboração industrial. Quatro comunidades de design estão envolvidas com os estudos do centro de pesquisa: estudantes, recém-formados, designers profissionais e professores acadêmicos, apoiando empresas, acadêmicos, governo e parceiros do setor voluntariado. Dentre os eventos organizados pelo CHHD está a Conferência Internacional sobre Design Inclusivo que, no ano de 2015, teve a sua oitava edição. O tema explorado nesta edição da Conferência foi "Rompantes inclusivos: ideias disruptivas em design inclusivo" ("Disruptive Inclusive: Disruptive ideas in inclusive design") (tradução nossa) que buscou reconhecer a difusão e a evolução do conceito e o cenário que ele tem se envolvido.

Ainda em Londres, se encontra também, a rede de pesquisa em design interdisciplinar e internacional - a Inclusive Design Research. Esta rede foi fundada em 2008 na Universidade de Brunel no oeste de Londres, se estendendo para a China em 2010, na Tongji University em Shanghai.

A rede de pesquisa Inclusive Design Research promove o DI através de pesquisas aplicadas e transferência de conhecimento para a área acadêmica. Os pesquisadores criam ferramentas e estratégias de ensino do DI nas universidades. Entre os projetos desenvolvidos estão o "Facilitando a compreensão ampla do design inclusivo" ("Facilitating wider uptake of inclusive design") (tradução livre) (2008-2011), atuando como pesquisadores principais e o "Aplicação dos princípios do design inclusivo em produtos de cozinha" ("Application of inclusive design principles to kitchen products" ) (tradução livre) (2010), atuando como supervisores do projeto.

\section{Estados Unidos}

Nos Estados Unidos, onde se originou o Design Universal e seus sete princípios, encontra-se o Center for Universal Design (CUD) na Escola de Design da Universidade do Estado da Carolina do Norte. Esta escola oferece desde cursos para usuários a cursos de pós-graduação, conferências e palestras. As atividades de educação e formação do CUD abrange todas as idades, muitas audiências, e várias modalidades. Os tópicos abordados nas atividades oferecidas incluem produtos, habitação, ambiente construído não residencial, códigos e padrões e design urbano. (CUD, 2008) 
Nas suas atividades de pesquisa estão incorporados estudos de investigação aplicados em fatores humanos e as necessidades do usuário, usabilidade de produtos e ambientes acessíveis e universalmente concebidos, e o impacto do Design Universal no cotidiano das pessoas. Os cursos, oficinas e apresentações são destinados a estudantes, advogados, construtores, designers, engenheiros, prestadores de serviços e agências governamentais. O CUD conduz seminários e workshops e também, desenvolve e realiza programas de treinamento. (CUD, 2008)

No estado da Carolina do Norte também está sediado o Ronald L. Mace Universal Design Institute. Este instituto é uma organização sem fins lucrativos dedicada a promover o conceito e a prática de design acessível e universal. $O$ instituto oferece assistência à concepção e desenvolvimento de projetos; trabalha com pesquisa aplicada para gerar soluções de projeto apropriadas para a mais ampla diversidade de usuários; avalia produtos e edifícios existentes; colabora com os construtores e os fabricantes no desenvolvimento de novas soluções de design; desenvolve e distribui publicações educacionais e materiais de instrução úteis para profissionais que desejam praticar o DU [DI] e realiza treinamentos para aumentar o conhecimento da aplicação do DU [DI]. (RL MACE UNIVERSAL DESIGN INSTITUTE, 2016)

$\mathrm{Na}$ cidade de Buffalo no estado de Nova York se encontra, também, o Centro de Design Inclusivo e Acesso Ambiental (IDeA) localizado na Universidade de Buffalo. $O$ Centro IDeA fomenta práticas de design centrado no usuário através de pesquisa, desenvolvimento, serviços, divulgação e atividades educativas. O principal objetivo do Centro é produzir conhecimento e ferramentas para aumentar a participação social de grupos como: pessoas com deficiência e gerações mais velhas. (Centro IDeA, 2015)

Nas atividades de pesquisa realizadas no Centro estão incluídas: revisões sistemáticas, pesquisa sobre fatores humano, estudos de usabilidade no campo e de laboratório, grupos de foco e estudos etnográficos. Dentre as atividades de desenvolvimento estão: projetos arquitetônicos, produtos, recursos de tecnologia da informação e desenvolvimento organizacional. Entre as atividades educacionais, o Centro IDeA oferece suporte a educadores e estudantes em seu ensino e estudo do DU através de um site chamado Universal Design Education. Neste, estão disponíveis publicações; materiais de instrução, tais como: planos de estudos dos cursos, projetos de curso, palestras, amostra de trabalhos. Há também a divulgação de eventos de curto prazo, como: workshops e seminários. (Centro IDeA, 2015)

Ainda no estado de Nova York no Burton Blatt Institute (BBI) da Syracuse University se localiza a Global Universal Design Commission (GUDC), uma corporação sem fins lucrativos criada para aumentar a compreensão e uso do DU [DI]. A Comissão estabeleceu categorias de membros que busca envolver diretamente universidades, governos, desenvolvedores, proprietários de edifícios, organizações de pessoas com deficiência, instituições financeiras, e outras partes interessadas. Seu intuito é incentivar a participação dos indivíduos, organizações e governos na busca por saber mais sobre $\mathrm{DI}$, além de acelerar o interesse, investimento, e adoção dessa prática.

A Comissão oferece, também, treinamentos e assistência técnica com o intuito de ajudar na compreensão e na aplicação normas do DU voltadas para a melhoria de produtos e ambiente construído. Além de fomentar o ensino e a pesquisa dessa abordagem de projeto, a GUDC certifica profissionais para serem qualificados no DU e certifica também ambientes e produtos. 
Na cidade de Madison, está localizado o Trace Research \& Development Center e faz parte do College of Engineering da University of Wisconsin-Madison. O Centro foi fundado em 1971 com o intuito de atender as necessidades de comunicação de pessoas com limitações atípicas, ele é um dos primeiros líderes no campo, conhecido como "comunicação aumentada" (augmentative communication) (tradução livre). Os trabalhos desenvolvidos pelo Centro são focados na acessibilidade, todavia, reuni esforços para promover também o DI.

\section{Espanha}

Em Barcelona na Espanha foi criada em 2001 a Design For All Foundation, uma organização internacional sem fins lucrativos. A Fundação visa fomentar a pesquisa, 0 desenvolvimento de conhecimento, a promoção, a aplicação e a divulgação do Design Para Todos (DPT) em empresas, organizações privadas e públicas de educação. Ela contribui para a troca de conhecimento e divulgação através de redes internacionais, são elas: I) Rede de Excelência - Cidades e Vilas para Todos (Network for Excellence Towns and Cities for All )(tradução livre); II) IDeALL - Integrando Design Para Todos em Living Labs (IDeALL - Integrating Design for All in Living Labs )(tradução nossa); III) Museu Para Todos (Museum For All) (tradução livre) e IV) Sociedade Para Todos (Society for All) (tradução livre).

\section{Japão}

Em Tóquio e em Fukushima, no Japão, foi sediada a Conferência Internacional sobre Design Universal, em sua quinta edição em 2014, onde reuniu pesquisadores e empresas familiarizadas com o assunto. A Conferência é organizada pela International Association for Universal Design (IAUD) e o tema principal desta conferência foi "Desenvolvimento globalizado do Desenho Universal - Rumo a Tóquio 2020 Jogos Olímpicos e Paraolímpicos". O principal objetivo desta Conferência foi transmitir, do Japão para o mundo, os resultados dos avanços que têm ocorrido em pesquisas e práticas relacionadas com o DU [DI], e também compartilhar informações e proceder a trocas interpessoais. (IAUD, 2015)

A International Association for Universal Design teve origem na Conferência Internacional sobre Design Universal do ano de 2002 no Japão. Dentre as atividades realizadas pela Associação está o fomento do desenvolvimento de projetos relacionados ao $\mathrm{DI}$, que buscam estabelecer as metas de promoção dessa abordagem de projeto, generalizar os conceitos do DI, compartilhar conhecimento e informação e disseminar a conscientização deste entre os membros e consumidores. A IAUD realiza projetos de investigação conjunta com diferentes empresas e indústrias, baseando-se no diálogo com os consumidores. Uma das importantes atividades da associação está a criação de padrões e diretrizes a serem utilizados para promover DI. (IAUD, 2015)

A IAUD está vinculada a organizações localizadas em diversos países para trocar informações e interagir regularmente, a fim de promover a familiarização com a prática do DI. O nome e a localização dessas organizações são: I) Reino Unido - Helen Hamlyn Centre, Royal College of Art ; II) Noruega - O Centro Norueguês de Design e Arquitetura; III) Alemanha - if Universal Design \& Service GmbH (ex-Universal Design GmbH); IV) Espanha - Fundação Design Para Todos; V) Índia - DJ Academy of Design; VI) EUA - Instituto de Design Centrado no Humano; VII) EUA - TRACE CENTER Universidade de Wisconsin-Madison; VIII) EUA - IDeA Center, Universidade Estadual de 
Nova York / Buffalo; IX) EUA - Centro de Design Universal, Universidade do Estado da Carolina do Norte.

\section{Noruega}

O Conselho de Design Norueguês, através do Innovation for All Programme, vem desde 2005 elaborando e fornecendo técnicas e métodos para facilitar a prática do DI, além de apresentar também bons exemplos para inspirar e motivar projetos inclusivos. O Conselho também oferece um workshop desde 2013. As atividades do workshop são focadas nas áreas de produtos inclusivos, serviços e soluções digitais. O público-alvo são designers, executivos que atuam tanto em empresas privadas quanto no setor público, bem como pesquisadores e consultorias de design e treinamentos empresariais. O intuito dessa oficina é demonstrar os benefícios, destacar questões críticas e apresentar o valor comercial da abordagem. (CDN, 2016)

O Conselho de Design Norueguês também organiza a European Business Conference - Innovation for All, que acontece a cada dois anos. Esta conferência tem o intuito de promover o DI como uma abordagem centrada no usuário para a inovação. A primeira Conferência aconteceu em 2008 e a última, até então, em 2014, ambas em Oslo, capital da Noruega.

\section{Alemanha}

Na Alemanha encontra-se o iF Universal Design \& Service $\mathrm{GmbH}$, uma organização intermediada pelo Industrie Forum design eV (IF eV) que foi fundado em 1953. Esta organização visa homenagear e divulgar exemplos de bons projetos inclusivos. Com cinco anos de execução, em fevereiro de 2016 aconteceu em Munique a recente exposição dos projetos premiados.

\section{Índia}

A DJ Academy of Design (DJAD), em associação com o British Council organizou uma conferência de dois dias sobre "Design Universal e Desenvolvimento" (Universal Design and Development) (Tradução livre). A conferência é um seguimento de estudos feitos em pareceria com professores do Reino Unido. Ela aconteceu em março de 2015, oferecendo três sub-temas: I) Consciência do Design Universal; II) Design Universal e Educação e III) Prática do Design Universal. Esta foi a primeira conferência internacional exclusivamente em DU realizada na Índia. A DJAD oferece cursos de pósgraduação de dois a três anos de duração e um deles é a especialização em Desenho Industrial que possibilita o foco de estudo em DU. A academia é localizada no estado de Tamil Nadu na Índia.

\section{Visão Geral}

Com o intuito de ter uma visão panorâmica da disseminação do Design Inclusivo no mundo, a seguir, a Figura 1 ilustra os locais onde há o fomento dessa prática. 


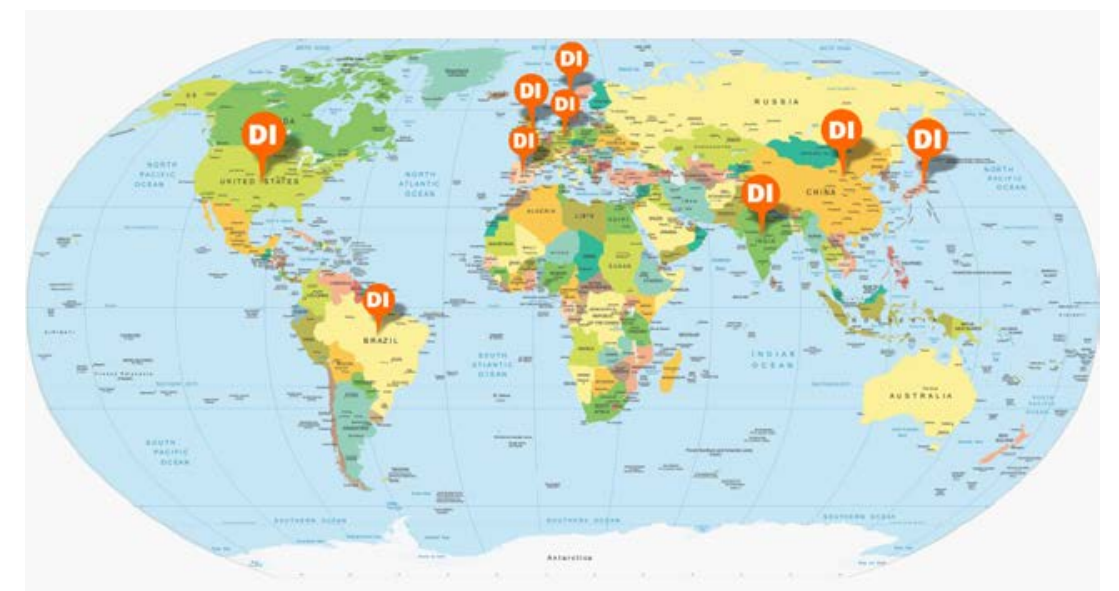

Figura 1 - Mapa Mundi mostrando as localidades onde o Design Inclusivo é fomentado.

Fonte: Elaborado pelo autor, com base na pesquisa realizada.

Tendo em vista o conteúdo levantado, percebe-se o fomento do DI em diversos locais do mundo. A pesquisa e o ensino caminham lado a lado, promovendo uma troca rica entre as diferentes realidades encontradas nos diversos países envolvidos com o assunto. Nota-se a forte presença do DI em países líderes de fabricação e potências econômicas como os Estados Unidos e o Japão. O reconhecimento dessa abordagem de projeto por países tão influentes mostra a importância do ensino e da prática para as sociedades desenvolvidas.

Um grande número de estudos e artigos publicados a respeito do DI no Reino Unido vem do Engineering Design Centre da Universidade de Cambridge e do Royal College of Art. Artigos relacionados ao assunto também são produzidos em outros países como a China, encontra-se publicações do Graduate Institute of Design Science, da Universidade de Tatung, localizada em Taiwan.

Vale ressaltar que o ensino do DI não se limita apenas aos centros de pesquisa e universidades citadas, pois há o fomento dessa prática de projeto em outros países, ainda que em pouca quantidade. O mesmo acontece com o Brasil, o desconhecimento não é total, mas ainda não é o suficiente para que o DI seja praticado com eficácia e produzido em grande escala pelos fabricantes.

Entendendo que a formação, a orientação e o conhecimento são fatores importantes na transposição do conceito do DI para a prática de projeto, o ensino é um desafio a ser travado não só no Brasil como em diversos outros países do mundo. Acredita-se que é preciso mais difusão do ensino para que mais profissionais fiquem cientes dessa prática transformadora.

\subsection{Prática Profissional}

A princípio, segundo alguns autores, dois principais fatores que dificultam a prática do DI são o tempo e o orçamento destinados ao projeto que, por sua natureza, exige pesquisa e conhecimento dos usuários. Dong et al. (2003) explicam que a teoria emergente do DI, influenciado pelo Design Centrado no Usuário (DCU), tende a enfatizar o envolvimento do usuário final no processo de concepção e avaliação iterativa desde o início, e continuando por toda parte, todo o processo de concepção do projeto. No entanto, tanto o envolvimento do usuário final e avaliação iterativa são demorados e, consequentemente, caros. Os autores ainda reforçam a restrição de tempo e a falta de avaliação prática de projeto com a necessidade de fazer 
lançamentos rápidos para o mercado. Como o processo de design é normalmente limitado pelo tempo e orçamento, pode não ser prático implementá-lo.

O fato é que há a necessidade de que os designers compreendam as características e capacidades dos usuários. Porém, estudos comprovam que raramente há o contato direto entre os designers e os usuários. Segundo Dong et al. (2003) esta situação não se restringe apenas à prática do DI, de maneira geral, ainda há uma distância entre designers e usuários, da mesma forma como o conhecimento do usuário final e sua real perspectiva. Os autores ainda afirmam que os usuários estão, geralmente, mais distantes dos designers do que dos clientes.

Segundo Kai-Chieh Lin \& Chih-Fu Wu (2015), durante o processo de concepção de produtos, estudos de mercado são realizados e os resultados são usados para determinar a orientação do projeto. Os autores afirmam ainda que a concepção dos produtos é muitas vezes baseada nos pensamentos subjetivos dos designers, e não convertem com precisão os pareceres da maioria dos usuários em fatores de design que podem servir como uma referência de projeto. Isto reforça a afirmação de Dong et al. (2003) quanto a distância entre as necessidades do usuário e o conhecimento que o designer tem dos mesmos.

O DCU é um conceito familiar para aqueles que trabalham com Fatores Humanos/Ergonomia e Usabilidade. É também familiar para aqueles que estudam o Design Inclusivo. Logicamente, se um produto ou serviço se destina a ser utilizado por pessoas com limitações funcionais deve satisfazer as suas necessidades (LAW et al., 2010). Embora DCU seja um termo familiar para muitos no campo, segundo Law et al. (2010) a prática deste é comumente ausente no desenvolvimento de produtos.

Diante da opinião de estudiosos sobre o envolvimento do usuário no processo de elaboração do produto, é válido considerar o contexto em que os estudos foram publicados. Cabe aqui levantar algumas questões: será que esta realidade, onde o envolvimento do usuário é raro nos projetos de produtos, ainda vigora nos tempos de hoje? Será que o tempo dedicado ao envolvimento do usuário é de fato um custo a mais em um projeto ao passo que este deveria ser indispensável em um projeto de Design?

Acredita-se que é cada vez mais frequente e necessário esse envolvimento, não obstante, ainda há a necessidade de se questionar a visão dos designers em seus projetos. Sendo o Design centrado no usuário, levanta-se a questão: e a diversidade humana? É neste ponto que acreditamos estar a verdadeira evolução da concepção de projeto de Design. Os custos de projeto não são necessariamente elevados por conta dessa abordagem (DI), partindo do pressuposto de que o Design é centrado no usuário e necessita do conhecimento amplo do seu público. Portanto, se o Design é centrado no humano, do mesmo modo, deve-se reconhecer a diversidade funcional que abarca o uso do objeto em projeto.

À vista disso, acredita-se que não há resultado factível, ou seja, projeto de sucesso sem o conhecimento do usuário no processo de elaboração. $O$ envolvimento do usuário, considerando o $\mathrm{DI}$, deve estar presente na abordagem raiz que prevê os passos do projeto, isto é, a otimização dos custos se configura no processo com o planejamento do mesmo.

Com relação aos custos de produção de produtos, Story et al. (1998) afirmam que o custo razoável é um problema encontrado em qualquer processo de design e produção. Portanto, os autores reforçam que o Design Universal [DI] tornou-se uma 
abordagem muito vendável, uma vez que aborda as diversas necessidades de uma gama maior de consumidores.

Subentende-se, que a capacidade do DI de reconhecer as diversas necessidades funcionais faz com que um produto garanta a eficácia de sua funcionalidade em diversos contextos de uso, portanto, é um produto que dispensa futuras adaptações, consequentemente, futuros gastos. Este é um fator significativo para que empresas compreendam que investir em produtos inclusivos evita futuras preocupações.

Outro fator importante levantado por Dong et al. (2003) é a pouca informação sobre as ferramentas e métodos de teste (usuário/produto) com as pessoas com deficiência. A ausência de métodos, técnicas e ferramentas para transpor o conceito do DI para a prática de projeto é um fator que dificulta a ação dos designers nesse processo. Law et al. (2010) atestam que os atuais recursos para tangibilizar o DU [DI], quanto a avaliação de sua real eficácia, são embrionários. Os autores afirmam que enquanto o conceito dessa prática de projeto está sendo explorado, ainda há muito a aprender sobre a forma como os recursos do DI são realmente utilizados.

Algumas ferramentas foram criadas para medir a exclusão que um produto pode ocasionar em um determinado público, ferramentas para avaliar o uso e a eficácia de um produto, e ainda, ferramentas de simulações de uso para que designers possam analisar as situações críticas de uso. Porém, até o momento não foram propagadas diretrizes que norteiem todo o processo de concepção, criação e avaliação de um produto inclusivo com comprovação de sua eficácia. Este pode ser um dos fatores responsáveis pela dificuldade em transpor a teoria do DI à prática de projeto.

\subsection{Barreiras entre o Design Inclusivo e a Indústria}

A dificuldade de aceitação por parte da indústria é um dos fatores que influenciam na ausência de produtos inclusivos no mercado. Essa dificuldade pode ocorrer por diversos motivos como: insegurança, recursos insuficientes, falta de estrutura necessária, dentre outros. Existem várias barreiras que impedem os fabricantes de enxergar e de produzir o DI. Essas barreiras muitas das vezes são de ordem equivocadas, ou seja, ideias preconcebidas sem fundamento; por vezes relacionadas a questões técnicas, isto é, dificuldades de prática (como vimos na sessão anterior), outrora, por questões relacionadas à natureza e cultura da empresa.

Dong et al. (2004) apresentam, no artigo Inclusive Design in Industry, estudos feitos com fabricantes e comerciantes no Reino Unido, no Japão e nos Estados Unidos. Os autores observaram que, no Reino Unido, a maioria das "barreiras de percepção" são classificadas como mais significativas pelos fabricantes, seguido por "barreiras técnicas" e depois "barreiras organizacionais". Os autores acreditam que isto indica que os esforços devam ser direcionados para a sensibilização com o intuito de superar as barreiras de percepção, ou seja, de esclarecimento do conceito e da prática do DI, bem como o investimento e os ganhos na produção de produtos inclusivos, além de fornecer ferramentas de apoio para superar as barreiras técnicas.

Nos Estados Unidos, através do projeto Universal Design Research Project(1998), foi identificada uma série de barreiras de fabricantes de produtos de consumo. Alguns fatores significativos que influenciam a não aplicação do Design Inclusivo, foram: I) a insegurança do investimento e a falta de interesse por conta da direção empresarial e estrutura da empresa não apropriada e II) a ausência de profissionais gabaritados para elaborarem projetos inclusivos. (DONG et al., 2004) 
Portanto, nas barreiras entre a aplicação do DI e a indústria dos Estados Unidos, destacam-se dois agentes: a diretoria da empresa, a qual apresenta a insegurança, o desinteresse e a desestruturação para adotar o DI; e o designer, o qual não está preparado para elaborar produtos inclusivos.

No Japão, através do projeto Kyoyo-hin (Design Universal) (2000) identificou-se alguns fatores relacionados a insegurança dos investidores bem como nos Estados Unidos e fatores relacionados a prática do designer, como a falta de conhecimento, técnicas e métodos foram determinantes (DONG et al., 2004). Portanto, observa-se que a dificuldade não está somente atrelada a prática, mas também ao conhecimento da teoria do DI. Dois agentes estão ligados a essas barreiras, tanto no Reino Unido como nos Estados Unidos e no Japão, os designers e a diretoria empresarial. 0 designer carrega, desse modo, um papel extremamente importante neste contexto. Entende-se que a iniciativa de aplicar o Design Inclusivo tem que partir deste profissional, visto o seu potencial em tornar produtos rentáveis e toda a sua bagagem de conhecimentos para transformar ideias em objetos. Mesmo que haja dificuldades, como uma pesquisa mais detalhada, avaliações participativas, dentre outras, o convencimento do investimento está nas mãos deste profissional.

\section{CONCLUSÃO}

É importante observar a preocupação dos institutos de pesquisa e das instituições de ensino que fomentam o DI nos países citados quanto a sua aplicação e a aceitação da indústria, criando ferramentas que auxiliam a prática, além de buscar também esclarecer os benefícios de negócios para as empresas que investirem em projetos inclusivos. Esta dedicação fica mais clara no Reino Unido, apesar de estar presente também em outros lugares.

Diante do exposto, percebe-se que há a necessidade da união de esforços, envolvendo os docentes dos cursos de formação, designers atuantes no mercado e diretores industriais. A inserção do DI deve se iniciar nas instituições de ensino para que profissionais saiam aptos nessa prática, mas é crucial que a indústria abrace essa filosofia, aberta à inovação e a novos negócios.

Retomando o que já foi discutido, é importante deixar claro que investir em produtos inclusivos pode ser um potencial de negócios abarcando um número maior de usuários, consequentemente, conquistando mais consumidores representativos. 0 ideal é mostrar para os fabricantes que o DI além de ser relevante para a inclusão social, ele é uma forma de conquistar mercados até então inexplorados e pode ser um caminho valioso para a inovação. Sendo assim, produtos excludentes podem ser vistos como uma lacuna na lista de produtos eficientes, enquanto produtos inclusivos podem ser considerados com um potencial de venda.

Acredita-se, em uma perspectiva otimista, que a maneira como o DI vem sendo trabalhado ao longo do tempo, com a visão mais clara dos potenciais mercados até então inexplorados bem como os recursos criados para facilitar a prática dessa abordagem de projeto, as barreiras entre o DI e a indústria tendem a exaurir.

Contudo, acredita-se que foi possível compreender o contexto dessa abordagem de projeto no mundo, não obstante, questões mais complexas como: "por que não se ensina?" e "por que não se aplica?" ainda permeiam o Brasil. Portanto, como já foi dito, uma investigação será feita com professores do curso de graduação 
em Design e profissionais da área com o intuito de responder tais perguntas e assim, ter uma visão mais clara do contexto do DI no Brasil.

\section{AGRADECIMENTOS}

Agradecimento especial ao Conselho Nacional de Desenvolvimento Científico e Tecnológico (CNPq) pelo apoio a pesquisa em desenvolvimento.

\section{REFERÊNCIAS}

DONG, H., Keates, S., Clarkson P. J.: Implementing Inclusive Design: the Discrepancy between Theory and Practice. In: Carbonell, N., Stephanidis, C., (eds.): Universal Access: Theoretical Perspectives, Practice, and Experience. 7th ERCIM International Workshop on User Interfaces for All, Paris, France, October 2002, Berlin Heidelberg New York; 2003 p.106-117

DONG, H., Keates, S., Clarkson P. J.: Inclusive Design in Industry: Barriers, Drivers and the Business Case. Berlin Heidelberg; 2004 p. 305-319

MONT'ALVÃO, C. R.; VILLAROUCO, V. Um novo olhar para o projeto: a ergonomia no ambiente construído. 1. ed. Teresopolis, RJ: 2AB, 2011. v. 1. 184p

LAW, Chris M.; Chris M. Law; Paul T. Jaeger; Elspeth McKay. User-centered design in universal design resources? Univ Access Inf Soc (2010) 9:327-335. Published online: 20 August 2010

LIN, Kai-Chieh.; Kai-Chieh Lin a; Chih-Fu Wu. Practicing universal design to actual hand tool design process. Applied Ergonomics 50 (2015) 8 a 18p

STORY, Molly. Molly Follette Story; James L. Mueller; Ronald L. Mace. The Universal Design File: Designing for People of All Ages and Abilities State. University, The Center for Universal Design, 1998.

Sites:

Center for Inclusive Design and Environmental Access (IDeA); Faculdade de Arquitetura e Planejamento. Nova York; Estados Unidos da América. Disponível na internet por http em: <http://idea.ap.buffalo.edu/> Acessado em: Novembro de 2015

Centre for Universal Design (CUD); Escola de Design da Universidade do Estado da Carolina do Norte; Carolina do Norte; Estados Unidos da América. Disponível na internet por http em: <https://www.ncsu.edu/ncsu/design/cud/index.htm> Acessado em: Novembro de 2015.

Centre Helen Hamlyn for Design (CHHD); Royal College of Art; Londres; Reino Unido. Disponível na internet por http em: <http://www.rca.ac.uk/research-innovation/helenhamlyn-centre/> Acessado em: Novembro de 2015.

Design for All Foundation; Barcelona, Espanha. Disponível na internet por http em: <http://designforall.org/> Acessado em: Novembro de 2015.

DJ Academy of Design. Tamil Nadu; Índia. Disponível na internet por http em: <http://www.djad.in/> Acessado em Junho de 2016.

if Universal Design \& Service GmbH. Munique; Alemanha. Disponível na internet por http em: <http://www.if-universaldesign.eu/index.html> Acessado em Junho de 2016. 
Inclusive Design Group; Engineering Design Centre. Disponível na internet por http em: <http://www.inclusivedesigngroup.com/> Acessado em: Novembro de 2015.

Inclusive Design Research; Londres; Reino Unidos. Disponível na internet por http em:< http://www.inclusivedesignresearch.org/ > Acessado em Abril de 2016.

International Association for Universal Design; Japão. Disponível na internet por http em: < http://www.iaud.net/> Acessado em: Novembro de 2015.

Global Universal Design Commission, Inc. (GUDC); Nova lorque; Estados Unidos da América. Disponível na internet por http em: <http://www.globaluniversaldesign.org/ > Acessado em Abril de 2016.

Norwegian Design Council; Oslo; Noruega. Disponível na internet por http em: <http://www.norskdesign.no/old_en/> Acessado em: Junho de 2016.

RL Mace Universal Design Institute; Carolina do Norte; Estados Unidos da América. Disponível na internet por http em: <http://www.udinstitute.org/> Acessado em: Abril de 2016.

Universidade de Cambridge. Cambridge; Reino Unido. Disponível na internet por http em: < https://www-edc.eng.cam.ac.uk/research/inclusivedesign/> Acessado em: Maio de 2016. 